\title{
Severe Infliximab-Induced Alopecia and Scalp Psoriasis in a Woman with Crohn's Disease: Dramatic Improvement after Drug Discontinuation and Treatment with Adjuvant Systemic and Topical Therapies
}

Jeremy Udkoff · Philip R. Cohen

Received: September 1, 2016 / Published online: November 14, 2016

(C) The Author(s) 2016. This article is published with open access at Springerlink.com

\section{ABSTRACT}

Scalp psoriasis with alopecia is a rare cutaneous reaction to tumor necrosis factor alpha antagonists. This reaction often reverses with discontinuation of the offending drug and initiation of topical treatments; however, irreversible hair loss may occur if a scarring alopecia develops. We describe a woman with Crohn's disease who developed scalp psoriasis and alopecia secondary to infliximab. She had a remarkable recovery after discontinuation of infliximab and treatment with oral minocycline and topical therapy: mineral oil under occlusion, betamethasone lotion, and sequential coal tar, salicylic acid, and ketoconazole shampoos each day. The patient's alopecia completely resolved within 4

Enhanced content To view enhanced content for this article go to www.medengine.com/Redeem/ 5617F060724804D6.

J. Udkoff $(\bowtie)$

Medical School, University of California San Diego,

San Diego, CA, USA

e-mail: jeremy.udkoff@gmail.com

P. R. Cohen $(\varangle)$

Department of Dermatology, University of

California San Diego, San Diego, CA, USA

e-mail: mitehead@gmail.com months of initiating this treatment regimen. In summary, early diagnosis of alopecia secondary to tumor necrosis factor alpha antagonist therapy is crucial in preventing diffuse alopecia and scalp psoriasis. In addition to discontinuing the offending agent, initiating aggressive adjuvant treatment with an oral antibiotic, topical therapies, or both, should be considered to reverse tumor necrosis factor alpha antagonist-induced alopecia and/or scalp psoriasis.

Keywords: Alopecia; Crohn's; IBD; Inflammatory bowel disease; Infliximab; Psoriasis; Scalp; Tumor necrosis factor alpha

\section{INTRODUCTION}

Tumor necrosis factor alpha antagonists can treat a variety of rheumatic diseases including ankylosing spondylitis, inflammatory bowel diseases, and psoriasis [1]. Yet, these drugs may paradoxically cause psoriasis or scalp alopecia, or both. Tumor necrosis factor alpha inhibitor-induced psoriasis is rare with an incidence of 1.04-3.0 cases per 1000 
person-years [2]. This may result in skin or scalp lesions. Rarely, a scarring alopecia may develop; this reaction has only been described twice in the literature-both in patients being treated with adalimumab $[3,4]$. We report a patient with Crohn's disease being treated with infliximab who developed alopecia and scalp psoriasis. The patient's scalp remarkably improved after discontinuation of infliximab and the initiation of oral and topical therapies. All procedures followed were in accordance with the ethical standards of the responsible committee on human experimentation (institutional and national) and with the Helsinki Declaration of 1964, as revised in 2013. Informed consent was obtained from the patient for being included in the study.

\section{CASE REPORT}

A 23-year-old female with a history of Crohn's disease had previously presented to another dermatologist for evaluation of alopecia. She had been treated with azathioprine, and her hair loss began 8 months after adding infliximab therapy.

At that time, her scalp examination showed diffuse alopecia and an erythematous plaque with superficial scaling (Fig. 1). A punch biopsy of the scalp was performed, and histopathologic examination revealed chronic folliculitis and perifolliculitis with dermal scarring and naked hair shafts in the dermal stroma. These histopathologic findings were consistent with a diagnosis of folliculitis decalvans.

Infliximab infusions were discontinued, and the patient was prescribed oral minocycline (100 mg twice daily) to treat her folliculitis decalvans. During the subsequent month, her alopecia progressed, and she developed pruritic lesions that covered her scalp. She presented to us for a second opinion.

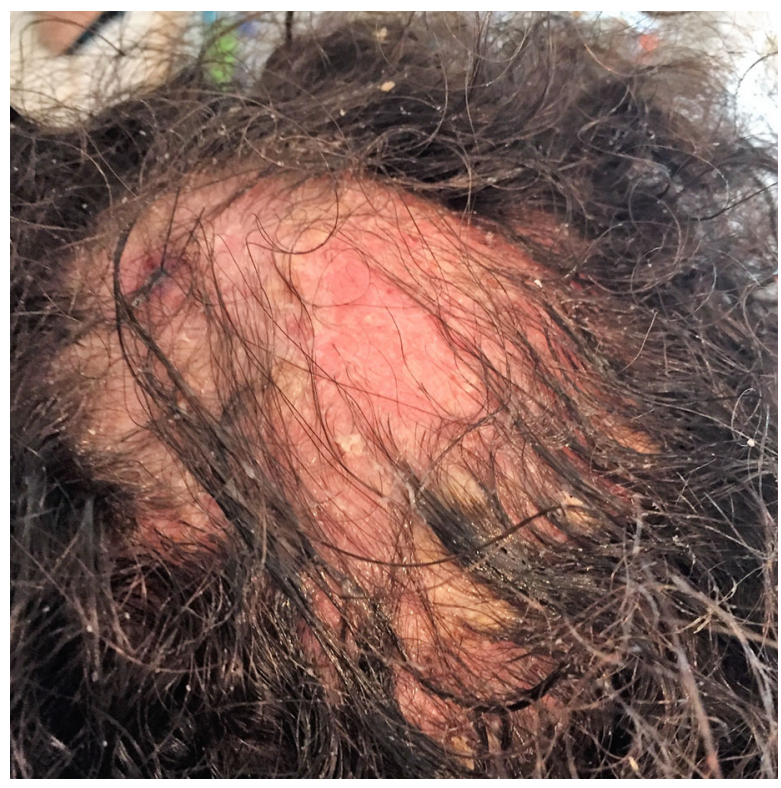

Fig. 1 The scalp of a 23-year-old female who had been receiving infliximab to treat Crohn's disease. An erythematous plaque with overlying scale and diffuse alopecia is noted

Her scalp examination now demonstrated psoriasiform changes characterized by well-defined erythematous plaques with an overlying white silvery scale (Fig. 2). The diagnosis of alopecia and scalp psoriasis secondary to infliximab treatment was suspected. Severe seborrheic dermatitis and/or scalp infection was also included in the differential diagnosis.

Initially, adjuvant topical interventions were initiated. The patient applied betamethasone lotion twice daily. In addition, mineral oil was covered with a shower cap on the scalp overnight. Each morning, either 5\% coal tar, $2 \%$ ketoconazole, or $6 \%$ salicylic acid shampoo was used to wash the scalp. The twice daily oral minocycline was maintained, and a bacterial culture of her scalp was performed.

Two additional bacterial cultures of her scalp (monthly, over the subsequent months) were performed to assess for the possibility of associated infection; the patient continued the 


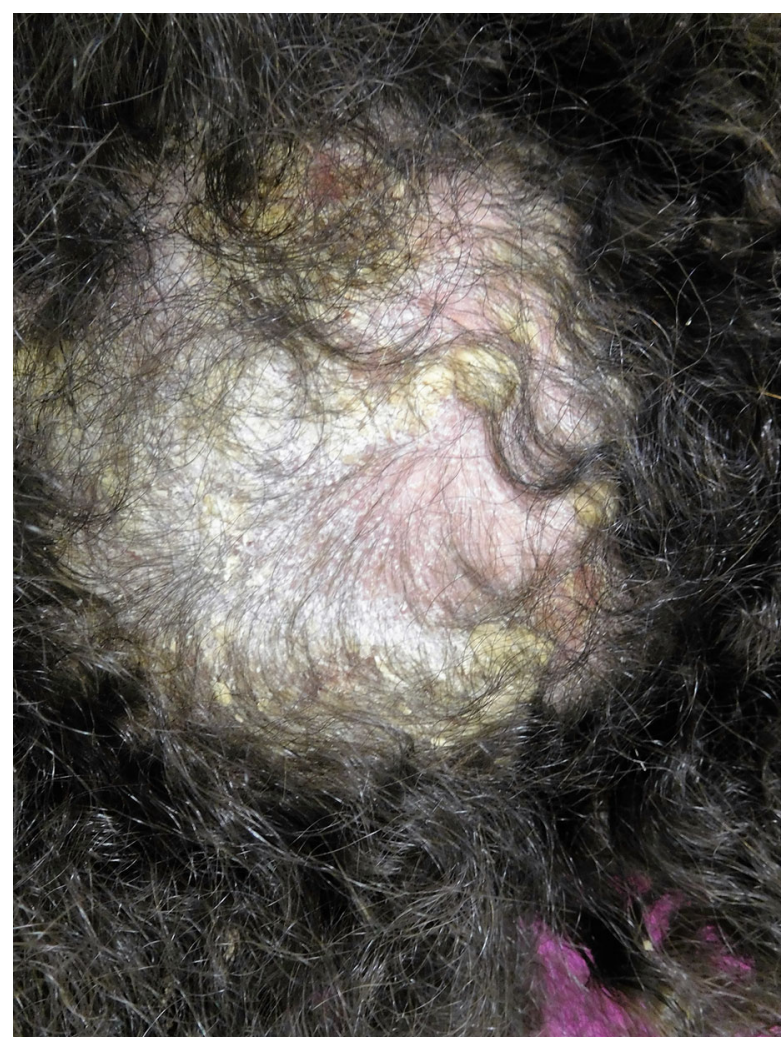

Fig. 2 The patient's scalp 1 month after beginning minocycline therapy and discontinuing infliximab. Her alopecia persists; in addition, psoriasiform changes characterized by a well-defined erythematous plaque with an overlying white silvery scale are present

oral minocycline therapy during this time. The scalp bacterial cultures grew Acinetobacter species, Acinetobacter baumanii, and Acinetobacter radioresistens, respectively-each of the organisms was susceptible to minocycline. However, since the variants of Acinetobacter continued to persist while she received minocycline treatment, consultation with an infectious disease specialist determined that the isolated bacteria were commensal species, and the oral antibiotic was discontinued.

The patient's alopecia and scalp psoriasis both remarkably improved. Indeed, at her 4-month follow-up visit there were no scalp scales and all of her hair had returned (Fig. 3).
Her topical scalp therapies were tapered and subsequently discontinued. Neither her alopecia nor scalp psoriasis recurred.

Ustekinumab was initiated to treat her Crohn's disease, and her inflammatory bowel disease remains well controlled. In addition, she has not developed either alopecia or scalp psoriasis.

\section{DISCUSSION}

Anti-tumor necrosis factor alpha therapy may be associated with alopecia, dermatitis, infections, and psoriasis [1, 5]. Rheumatoid arthritis is the most common underlying illness, present in $46.0 \%(80 / 174)$ of patients, associated with tumor necrosis factor alpha antagonist-induced psoriasis [6]. This adverse event usually manifests as a pustular eruption on the palms of the hands and/or soles of the feet (palmoplantar pustulosis). Palmoplantar pustulosis is very rare within the general population, so its discovery should prompt investigation of the patient's medications [6].

A retrospective analysis found 29.4\% (203/ 690) of Crohn's patients who began tumor necrosis factor alpha-antagonist therapy developed various skin lesions [7]. Psoriasis composes $22.9 \%(8 / 35)$ of anti-tumor necrosis factor alpha-related cutaneous reactions with a prevalence of $1.5-5 \%$ in these patients $[1,8]$. It may occur days to years after beginning treatment, and women from 40 to 50 years old are at the highest risk of developing a psoriasiform reaction $[6,8]$.

Scalp psoriasis is usually characterized by discrete psoriatic plaques on the scalp, with or without alopecia, that may itch intensely $[3,4,9,10]$. In contrast to palmoplantar pustulosis (where rheumatoid arthritis is the most common underlying disease), Crohn's disease usually underlies the very rare 

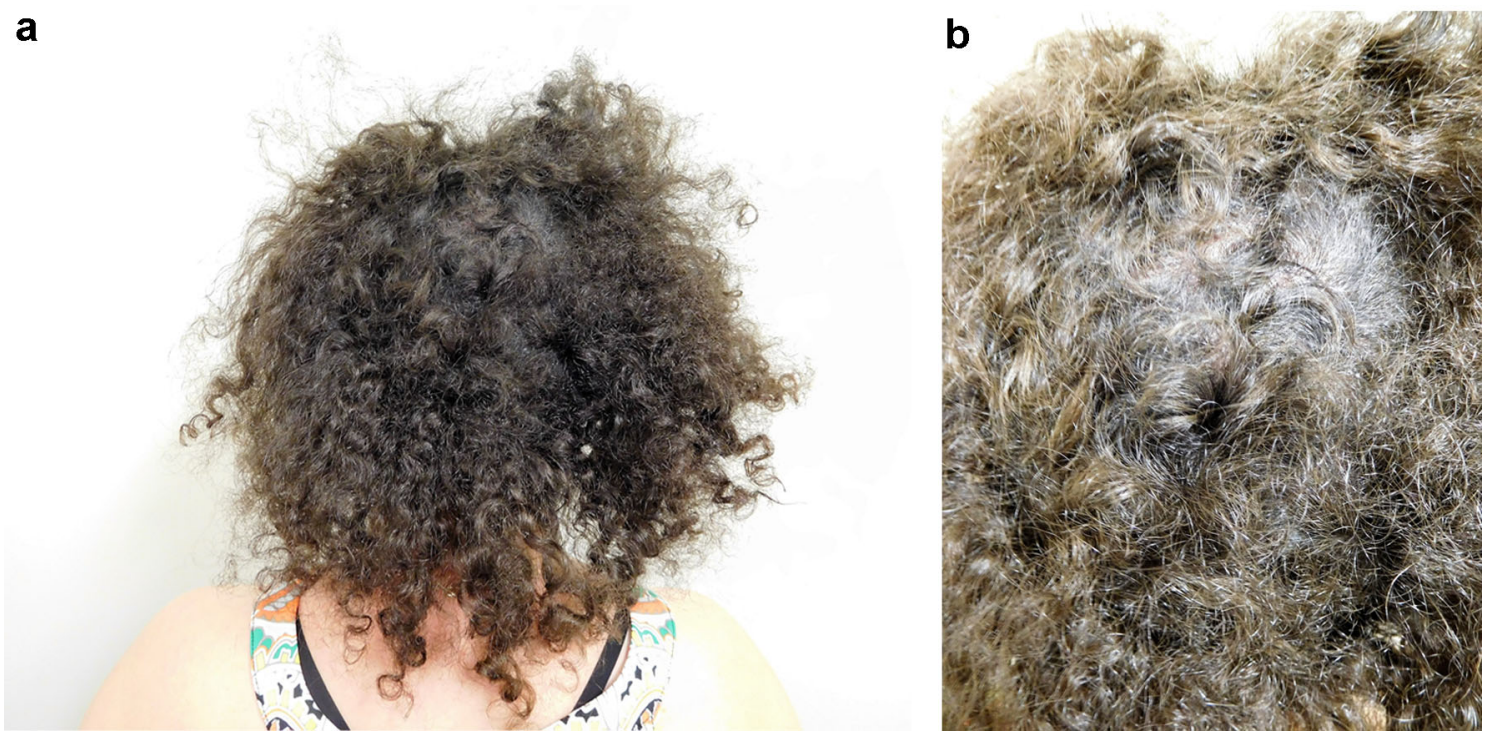

Fig. 3 Distant (a) and closer (b) images of the patient's hair and scalp. The patient's alopecia had completely resolved at her 4-month follow-up visit (a). Small areas with shorter hair, representing new hair growth, are seen $(\mathbf{b})$

manifestation of tumor necrosis factor alpha antagonist-induced scalp psoriasis. A case series and literature review reported 15 such individuals; $80 \%(12 / 15)$ of the patients had a history of Crohn's disease [10].

The differential diagnosis of tumor necrosis factor alpha antagonist-induced hair loss includes alopecia areata, pityriasis amiantacea, psoriasis, and tinea capitis [11]. A retrospective review of drug reactions found infliximab to be the most common tumor necrosis factor alpha-antagonist to cause alopecia (18/52 cases, 35\%) [12]. This study also noted that tumor necrosis factor alpha-antagonist exposure was three times more likely (reporting odds ratio $=3.0$ ) in patients with alopecia than other adverse drug reactions. Another study found the average duration of exposure to tumor necrosis factor alpha-antagonists before the development of alopecia areata to be 22.5 months [13]. This prospective report found $76 \%$ of alopecia areata cases to partially or completely resolve over a mean of 5 months with no difference in alopecia areata resolution between those that discontinued and continued tumor necrosis factor alpha-antagonist treatment.

Biopsy and histologic examination of the alopecia may be useful in establishing the diagnosis. In addition to classic psoriasiform epidermal changes of idiopathic psoriasis, the histopathology of tumor necrosis factor alpha antagonist-induced psoriasis can also show alterations similar to alopecia areata. These may include hair follicle miniaturization, increased catagen and telogen hairs, and peribulbar lymphocytic infiltrate occupying all levels of the terminal hair structure [11].

The histology of our patient's scalp alopecia (consisting of chronic folliculitis and perifolliculitis, dermal scarring, and naked hair shafts in the dermal stroma) was consistent with the diagnosis of folliculitis decalvans-a type of scarring alopecia that may present with pustules, inflammatory papules, tunneling hairs, and permanent hair loss [14]. The dramatic response to our intervention with complete regrowth of hair was unexpected 
based on this presumed diagnosis. Furthermore, infliximab has been used to treat folliculitis decalvans, so its discontinuation would not be expected to cure the disease [15].

Therefore, in our patient, it is possible that a reversible scarring alopecia resembling folliculitis decalvans concurrently occurred with psoriasis scalp. Alternatively, it is possible that the biopsy was obtained from a non-representative area of the scalp, and the true diagnosis was tumor necrosis factor alpha-induced psoriasis complicated by an overlying cellulitis. Minocycline was initially selected as a first-line therapy for folliculitis decalvans [16]. However, it likely aided in resolving an overlying cellulitis or impetiginization and may have helped to decrease local dermal inflammation.

Infliximab may be used to treat psoriasis. Yet, paradoxically, infliximab is implicated in the development of this very disease especially in patients with Crohn's disease $[6,15,17]$. In vitro tumor necrosis factor alpha significantly inhibits hair growth [18]. However, tumor necrosis factor alpha antagonists may cause hair loss instead of inhibiting it.

A possible mechanism for tumor necrosis factor alpha antagonist-induced psoriasis involves dermal plasmocytoid dendritic cells. These cells produce interferon-alpha, an important cytokine in the pathogenesis of psoriasis, and may be induced by tumor necrosis factor alpha antagonists. In turn, this would cause the migration of $\mathrm{T}$ cells to the skin via interleukin-15 and a psoriasiform reaction [8]. It is possible that alopecia and psoriasis occurred in the patient secondary to the anti-tumor necrosis factor alpha agent as these diseases seem to be related to different aspects of the medication.
In tumor necrosis factor alpha antagonist-induced psoriasis, the offending drug should be discontinued and topical corticosteroid therapy should be initiated. In one review, $82 \%(50 / 61)$ of patients experienced complete resolution of their drug-induced psoriasis after discontinuing the offending tumor necrosis factor alpha antagonist. However, many patients cannot discontinue their tumor necrosis factor alpha antagonist; most of these patients experience complete $(50 \%, 53 / 107)$ or partial $(49 \%, 52 / 107)$ resolution of their drug-induced psoriasis. Alternatively, a different tumor necrosis factor alpha antagonist may be attempted, and 52\% $(13 / 25)$ of patients had no reoccurrence of their lesions following this change [19].

If scalp psoriasis continues without treatment, permanent alopecia may result [10]. Topical corticosteroids, such as betamethasone $0.05 \%$ lotion and a daily shampoo with alternating agents $(5 \%$ coal tar, $2 \%$ ketoconazole, and $6 \%$ salicylic acid) that are applied to the scalp can be used in the treatment of scalp psoriasis [11]. In addition, mineral oil followed by occlusion with a shower cap can promote the desquamation of the plaques. Our patient's scalp was very sensitive; therefore, we used a corticosteroid in a lotion instead of a solution.

A recent report described the efficacy of using ustekinumab, an anti-interleukin-12/-23 monoclonal antibody, to treat concurrent inflammatory bowel disease and plaque psoriasis [9]. Our patient was also placed on ustekinumab to manage her Crohn's disease. However, her scalp condition had significantly improved prior to beginning the treatment. She is doing well on this therapy and has had no recurrence of her alopecia or scalp psoriasis. 


\section{CONCLUSION}

Tumor necrosis factor alpha antagonists may cause a variety of cutaneous adverse events. An idiosyncratic reaction of these drugs is the development of psoriasis-a condition they are used to treat. Although this reaction typically manifests as palmoplantar pustulosis, scalp psoriasis may also occur. Tumor necrosis factor alpha-induced scalp psoriasis appears to manifest most frequently in patients with Crohn's disease. Alopecia and possibly folliculitis decalvans may also be a sequella of this drug reaction. Thus, in addition to adjuvant measures to resolve the alopecia, psoriasis, or both-such as topical corticosteroids and therapeutic shampoosdiscontinuation of the offending drug and initiation of a different tumor necrosis factor alpha antagonist or immunotherapy, such as ustekinumab, is recommended. Minocycline treatment likely had a favorable influence on our patient's alopecia and scalp psoriasisperhaps secondary to the resolution of an overlying cellulitis or impetiginization, or as a result of its anti-inflammatory activity.

In summary, accurate diagnosis and treatment of infliximab-induced alopecia and scalp psoriasis resulted in an excellent clinical response for our patient with complete regrowth of her scalp hair. She was subsequently placed on an alternate inflammatory bowel disease therapy. Alopecia or psoriasis, or both, in a patient receiving infliximab, should prompt immediate discontinuation of the drug to prevent progression of the adverse cutaneous drug reaction.

\section{ACKNOWLEDGEMENTS}

No funding and no sponsorship were received in relation to this paper. All named authors meet the International Committee of Medical Journal Editors (ICMJE) criteria for authorship for this manuscript, take responsibility for the integrity of the work as a whole, and have given final approval for the version to be published.

Disclosures. Jeremy Udkoff, MA, and Philip R. Cohen, MD, have nothing to disclose.

Compliance with Ethics Guidelines. All procedures followed were in accordance with the ethical standards of the responsible committee on human experimentation (institutional and national) and with the Helsinki Declaration of 1964, as revised in 2013. Informed consent was obtained from the patient for being included in the study.

Open Access. This article is distributed under the terms of the Creative Commons Attribution-NonCommercial 4.0 International License (http://creativecommons.org/licenses/ by-nc/4.0/), which permits any noncommercial use, distribution, and reproduction in any medium, provided you give appropriate credit to the original author(s) and the source, provide a link to the Creative Commons license, and indicate if changes were made.

\section{REFERENCES}

1. Lee H-H, Song I-H, Friedrich M, Gauliard A, Detert J, Röwert J, Audring H, Kary S, Burmester G-R, Sterry W, Worm M. Cutaneous side-effects in patients with rheumatic diseases during application of tumour necrosis factor-alpha antagonists. $\mathrm{Br} \mathrm{J}$ Dermatol. 2007;156(3):486-91.

2. Cullen G, Kroshinsky D, Cheifetz AS, Korzenik JR. Psoriasis associated with anti-tumour necrosis factor therapy in inflammatory bowel disease: a new series and a review of 120 cases from the literature. Aliment Pharmacol Ther. 2011;34(11-12):1318-27.

3. Perman MJ, Lovell DJ, Denson LA, Farrell MK, Lucky AW. Five cases of anti-tumor necrosis factor 
alpha-induced psoriasis presenting with severe scalp involvement in children. Pediatr Dermatol. 2012;29(4):454-9.

4. El Shabrawi-Caelen L, La Placa M, Vincenzi C, Haidn T, Muellegger R, Tosti A. Adalimumab-induced psoriasis of the scalp with diffuse alopecia: a severe potentially irreversible cutaneous side effect of TNF-alpha blockers. Inflamm Bowel Dis. 2010;16(2):182-3.

5. Beccastrini E, Squatrito D, Emmi G, Fabbri P, Emmi L. Alopecia areata universalis during off-label treatment with infliximab in a patient with Behçet disease. Dermatol Online J. 2010;16(9):15.

6. Joyau C, Veyrac G, Dixneuf V, Jolliet P. Anti-tumour necrosis factor alpha therapy and increased risk of de novo psoriasis: is it really a paradoxical side effect? Clin Exp Rheumatol 2012;30(5):700-6.

7. Cleynen I, Van Moerkercke W, Billiet T, Vandecandelaere P, Vande Casteele N, Breynaert C, Ballet V, Ferrante M, Noman M, Van Assche G, Rutgeerts P, van den Oord JJ, Gils A, Segaert S, Vermeire S. Characteristics of skin lesions associated with anti-tumor necrosis factor therapy in patients with inflammatory bowel disease: a cohort study. Ann Intern Med. 2016;164(1):10-22.

8. Iborra M, Beltrán B, Bastida G, Aguas M, Nos P. Infliximab and adalimumab-induced psoriasis in Crohn's disease: a paradoxical side effect. J Crohns Colitis. 2011;5(2):157-61.

9. Andrisani G, Marzo M, Celleno L, Guidi L, Papa A, Gasbarrini A, Armuzzi A. Development of psoriasis scalp with alopecia during treatment of Crohn's disease with infliximab and rapid response to both diseases to ustekinumab. Eur Rev Med Pharmacol Sci. 2013;17(20):2831-6.

10. Osório F, Magro F, Lisboa C, Lopes S, Macedo G, Bettencourt H, Azevedo F, Magina S. Anti-TNF-alpha induced psoriasiform eruptions with severe scalp involvement and alopecia: report of five cases and review of the literature. Dermatology. 2012;225(2):163-7.

11. Ribeiro LBP, Rego JCG, Estrada BD, Bastos PR, Piñeiro Maceira JM, Sodré CT. Alopecia secondary to anti-tumor necrosis factor-alpha therapy. An Bras Dermatol. 2015;90(2):232-5.
12. Béné J, Moulis G, Auffret M, Lefevre G, Coquerelle P, Coupe P, Péré P, Gautier S. Alopecia induced by tumour necrosis factor-alpha antagonists: description of 52 cases and disproportionality analysis in a nationwide pharmacovigilance database. Rheumatology (Oxford). 2014;53(8):1465-9.

13. Tauber M, Buche S, Reygagne P, Berthelot J-M, Aubin F, Ghislain P-D, Cohen J-D, Coquerelle P, Goujon E, Jullien D, Brixi H, Jeudy G, Guennoc X, Martin A, Brénaut E, Hoppé E, Bertolotti A, Bardin T, Delaporte E, Allez M, Bachelez H, Seneschal J, Viguier M. Groupe de Recherche sur Psoriasis de Société Française de D, Club Rhumatismes et Inflammation (CRI), Groupe d'études thérapeutiques des affections inflammatoires du tube digestif (GETAID). Alopecia areata occurring during anti-TNF therapy: a national multicenter prospective study. J Am Acad Dermatol. 2014;70(6):1146-9.

14. Tan E, Martinka M, Ball N, Shapiro J. Primary cicatricial alopecias: clinicopathology of 112 cases. J Am Acad Dermatol. 2004;50(1):25-32.

15. Mihaljević N, von den Driesch P. Successful use of infliximab in a patient with recalcitrant folliculitis decalvans. J Dtsch Dermatol Ges. 2012;10(8):589-90.

16. Sillani C, Bin Z, Ying Z, Zeming C, Jian Y, Xingqi Z. Effective treatment of folliculitis decalvans using selected antimicrobial agents. Int J Trichology. 2010;2(1):20-3.

17. Loh TY, Cohen PR. Infliximab-associated psoriasiform dermatitis: case report and review of a seemingly paradoxical inflammatory response. Cureus. 2016;8(9):e773.

18. Philpott MP, Sanders DA, Bowen J, Kealey T. Effects of interleukins, colony-stimulating factor and tumour necrosis factor on human hair follicle growth in vitro: a possible role for interleukin-1 and tumour necrosis factor-alpha in alopecia areata. Br J Dermatol. 1996;135(6):942-8.

19. Collamer AN, Battafarano DF. Psoriatic skin lesions induced by tumor necrosis factor antagonist therapy: clinical features and possible immunopathogenesis. Semin Arthritis Rheum. 2010;40(3):233-40. 